\title{
A NUMERICAL STUDY OF DISSIPATIVE CHEMICALLY REACTIVE RADIATIVE MHD FLOW PAST A VERTICAL CONE WITH NON- UNIFORM MASS FLUX
}

\author{
P. SAMBATH \\ Department of Mathematics, S R M Institute of Science and Technology \\ Kattankulathur, Tamil Nadu-603203 INDIA \\ E-mail: sampathp@srmist.edu.in \\ D.S. SANKAR \\ School of Applied Sciences and Mathematics \\ Universiti Teknologi Brunei, JalanTungku Link \\ Gadong BE1410, Brunei Darussalam \\ E-mail: duraisamy.sankar@utb.edu.bn \\ K.K. VISWANATHAN* \\ Department of Basic Sciences and Mathematics \\ Kuwait College of Science and Technology \\ Doha District, Block 4, P.O. Box No. 27235, Safat 13133, KUWAIT \\ E-mail: visu20@yahoo.com; k.viswanathan@kcst.edu.kw
}

\begin{abstract}
A computational model is presented to explore the properties of heat source, chemically reacting radiative, viscous dissipative MHD flow of an incompressible viscous fluid past an upright cone under inhomogeneous mass flux. A numerical study has been carried out to explore the mass flux features with the help of CrankNicolson finite difference scheme. This investigation reveals the influence of distinct significant parameters and the obtained outputs for the transient momentum, temperature and concentration distribution near the boundary layer is discussed and portrayed graphically for the active parameters such as the Schmidt number Sc, thermal radiation $R d$, viscous dissipation parameter $\varepsilon$, chemical reaction parameter $\lambda$, MHD parameter $M$ and heat generation parameter $\Delta$. The significant effect of parameters on shear stress, heat and mass transfer rates are also illustrated.
\end{abstract}

Key words: free convection, finite difference, vertical cone, MHD, thermal radiation, viscous dissipation.

\section{Introduction}

The occurrence of mass transfer commonly exists in numerous chemical processing industries for instance food processing and polymer process.Magneto hydrodynamics is the science, which deals with the motion of an electrically conducting fluid in the presence of magnetic fields. The study of magneto hydrodynamics with heat and mass transfer in the presence of radiation has attracted many researchers and engineers due to diverse applications in different areas of science and technology. MHD plays an important role in geophysics, agriculture, petroleum industries, in exploration and thermal recovery of oil, geothermal reservoirs and underground nuclear waste storage sites. In the field of power generation, MHD is receiving

\footnotetext{
* To whom correspondence should be addressed
} 
considerable attention due to the possibilities of much higher thermal efficiencies in power plants. It is worth mentioning that the basic solution of thermal convection from a heated or cooled surface with suction or injection has numerous practical applications ranging from cooling of manufactured products to local weather forecasting.

The consequences of viscous dissipation takes a vital part in free convection for the range of applications that are subject matter to huge amount of deceleration or which functions at high rotational speeds. Analyzing the temperature and heat transfer have enormous significance to engineers since its universal incidence in several branches of engineering and science.

Rao and Shivaiah [1] presented a finite element method solutions for the governing equations under the influence of chemical reaction on a transient (MHD) flow from a semi infinite vertical plate with viscous dissipation. Siddiqa et al. [2] studied the natural convection flow of a viscous fluid above a semi-infinite horizontal plate with internal heat generation and variable viscosity. Srinivasa and Easwara [3] emphasised the transient mixed convection flow over a vertical plate moving in a parallel free flow with heat generation and absorption. Ramli et al. [4] examined the MHD convection flow due to external forces and transfer of heat by ferrofluids above a moving plate with consistent heat flux. Lakshmi Narayana and Sibanda [5] showed the Soret and Dufour effects on a natural convection over a vertical wavy surface in a Darcy porous medium. Kumari and Nath [6] investigated the natural convection effects over a flat cone under porous medium with VWT/VWC or VHF/VMF and heat source/sink. Pal and Talukdar [7] used perturbation technique for transient magneto hydro dynamic mixed convection flow in a chemically reactive, radiative micro polar fluid and Ullaha et al. [8] studied the above said effects with Maxwell fluid by homotopy analysis method.

A great number of engineering applications takes place at elevated temperatures and hence the knowledge of radiation and heat transfer is necessary for framing various models of industrial apparatus. Large amount of heat is generated when chemical reaction takes place in a process and because of this the temperature of the body increases. Several findings have been accounted on the mass transfer movement of a radiative fluids in the occurrence of MHD was studied by Sambath and Bapuji [9-12]. Muhaimin et al. [13] studied variable viscosity with thermally stratified impacts on magneto hydrodynamic free convective mass transfer over a porous wedge in the existence of chemical reaction. Zueco [14] presented the mixed convection mass transfer flow along a porous plate. Devi and Kumarai [15] discussed numerically the slip flow impacts on transient hydro magnetic flow over a enlarged exterior with thermal energy. Alam et al. [16] explored the analysis of transient magneto hydro dynamic natural convective flow of the heat transfer over a vertical porous flat plate with internal heat source. The consequence of double diffusion from a vertical truncated cone in a non-Newtonian fluid saturated porous medium with variable heat and mass fluxes wasaddressed by Awad et al. [17]. Cheng [18] executed the impact of MHD free convection flow with heat source in transient state. Khan et al. [19] examined the consequences of the transfer of heat energy flow of ferrofluids over a horizontal plate with consistent heat flux. Das et al. [20] attempted MHD free convective flow of nanofluids past a permeable plate in a rotating system with thermal energy. Also researchers [21-23] portrayed the impact of chemical reaction, MHD with stagnation point flow in porous medium. Chamka et al. [24] studied non-Darcy natural convective flow of nanofluid over a cone in a saturated permeable medium with homogeneous mass fraction flux. Ishak et al. [25] focused attention with similarity solutions for a vertical plate.

Recently the following investigators [27-33] presented electrically conducting, magneto hydrodynamic transfer of heat in a specific medium like Maxwell fluid, micro polar, nanofluid, Maxwell nanofluid with or without viscous dissipation, heat generation, absorption and mass flux. Magneto hydrodynamic convection flow due to the impacts of external forces and heat transfer over ferrofluids along a moving plate with uniform heat flux and second-order slip effects, chemical reaction with MHD stagnation point flow in porous medium were presented by the researchers [36-39].

In this study, the predominant idea is to simulate the numerical solution to radiative, chemically reactive, viscous dissipative MHD flow past a non- isothermal vertical cone in the occurrence of mass flux and heat generation by Crank-Nicholson scheme [26]. Such a thought is noteworthy in the research fields of biology and chemical engineering. The impacts of dissimilar relevant parameters are analyzed and the concluding results are demonstrated through graphs. 


\section{Mathematical formulation}

In this article, an axi-symmetric, transient boundary layer flow of a viscous incompressible electrically conducting fluid past a vertical cone with inconsistent heat mass fluxes under the influence of chemical reaction, heat generation under steady magnetic field $B_{0}$, dissipation effects. The flow is chosen along the direction of the $x$-coordinate and is chosen along the exterior surface of the cone in the vertical direction and the $y$ axis considered normally outward as shown in Fig.1. Since the outer surface is vast so the fluid flow extended towards infinity. The fluid taken for consideration being gray, absorbing-emitting but non- scattering medium because of this fact Rosseland approximation is employed to illustrate the radiative heat flux. Initially, when time $t^{\prime}<0$ the fluid as well as the outer surface of the cone is at rest with temperature $T_{w}^{\prime}$ and concentration $C_{w}^{\prime}$ at the wall and which is greater than the ambient temperature $T_{\infty}^{\prime}$ and ambient concentration $C_{\infty}^{\prime}$. When time $t^{\prime}>0$ the heat energy distributed from the exterior surface of the cone to the fluid medium at the rate of $q_{w}(x)=a x^{m}, q_{w}^{*}(x)=a x^{n}$ and is retained at this level. With these preceding assumptions, the equations which governs the flow, continuity, momentum, energy and concentration with the help of Boussinesq approximation can be written in a Cartesian form as mentioned below

$$
\begin{aligned}
& \frac{\partial}{\partial x}(u r)+\frac{\partial}{\partial y}(v r)=0 \\
& \frac{\partial u}{\partial t^{\prime}}+u \frac{\partial u}{\partial x}+v \frac{\partial u}{\partial y}-g \beta\left(T^{\prime}-T_{\infty}^{\prime}\right) \cos \phi-v \frac{\partial^{2} u}{\partial y^{2}}-g \beta_{c}\left(C^{\prime}-C_{\infty}^{\prime}\right) \cos \phi+\frac{\sigma B_{0}^{2} u}{\rho}=0 \\
& \rho C_{p}\left[\frac{\partial T^{\prime}}{\partial t^{\prime}}+u \frac{\partial T^{\prime}}{\partial x}+v \frac{\partial T^{\prime}}{\partial y}-\alpha \frac{\partial^{2} T^{\prime}}{\partial y^{2}}\right]=Q_{o}\left(T^{\prime}-T_{\infty}^{\prime}\right)-\frac{\partial q_{r}}{\partial y}-\mu\left(\frac{\partial u}{\partial y}\right)^{2} \\
& \frac{\partial C^{\prime}}{\partial t^{\prime}}+u \frac{\partial C^{\prime}}{\partial x}+v \frac{\partial C^{\prime}}{\partial y}-D \frac{\partial^{2} C^{\prime}}{\partial y^{2}}+k_{1}\left(C^{\prime}-C_{\infty}^{\prime}\right)=0
\end{aligned}
$$

The significant initial and boundary conditions are given by

$$
\begin{aligned}
& t^{\prime} \leq 0: u=0, \quad v=0, \quad T^{\prime}=T_{\infty}^{\prime}, \quad C^{\prime}=C_{\infty}^{\prime} \quad \text { for all } \quad x \quad \text { and } y, \\
& t^{\prime}>0: u=0, \quad v=0, \quad \frac{\partial T^{\prime}}{\partial y}=\frac{-q_{w}(x)}{k}, \quad \frac{\partial C^{\prime}}{\partial y}=\frac{-q_{w}^{*}(x)}{D}, \quad \text { at } \quad y=0, \\
& u=0, \quad T^{\prime}=T_{\infty}^{\prime}, \quad C^{\prime}=C_{\infty}^{\prime} \quad \text { at } \quad x=0, \\
& u \rightarrow 0, \quad T^{\prime} \rightarrow T_{\infty}^{\prime}, \quad C^{\prime} \rightarrow C_{\infty}^{\prime} \quad \text { as } \quad y \rightarrow \infty .
\end{aligned}
$$

The radiative heat flux expression $\frac{\partial q_{r}}{\partial y}$ appearing in the energy equation inthe $y$ - direction is made simpler by utilizing the Rosseland approximation given by Brewster [40]

$$
q_{r}=\frac{-4 \sigma^{*}}{3 k^{*}} \frac{\partial T^{\prime 4}}{\partial y} .
$$


Since the temperature variation within the flow is sufficiently small, we ignore higher order terms more than the first degree in $T^{\prime}-T_{\infty}^{\prime}$. The linear structure of Eq.(2.6) could be attained by escalating $T^{\prime 4}$ into Taylor series about $T_{\infty}^{\prime}$.

We obtain $\quad T^{\prime 4} \approx T_{\infty}^{\prime 3} T^{\prime}-3 T_{\infty}^{\prime 4}$.

Applying Eqs (2.5) and (2.6) in Eq.(2.3), we have

$$
\frac{\partial T^{\prime}}{\partial t^{\prime}}+u \frac{\partial T^{\prime}}{\partial x}+v \frac{\partial T^{\prime}}{\partial y}=\alpha \frac{\partial^{2} T^{\prime}}{\partial y^{2}}+\frac{Q_{O}}{\rho c_{p}}\left(T^{\prime}-T_{\infty}^{\prime}\right)-\frac{1}{\rho c_{p}} \frac{16 \sigma^{*} T_{\infty}^{\prime 3}}{3 k^{*}} \frac{\partial^{2} T^{\prime}}{\partial y^{2}}
$$

The local skin friction, local Nusselt number and local Sherwood number are

$$
\begin{gathered}
\tau_{x}=\left(\mu \frac{\partial u}{\partial y}\right)_{y=0} \\
\mathrm{Nu}_{x}=\frac{-\left(x \frac{\partial T^{\prime}}{\partial y}\right)_{y=0}}{T_{w}^{\prime}-T_{\infty}^{\prime}}, \\
\mathrm{Sh}_{x}=\frac{-\left(x \frac{\partial C^{\prime}}{\partial y}\right)_{y=0}}{C_{w}^{\prime}-C_{\infty}^{\prime}} .
\end{gathered}
$$

The time dependence assessments of skin friction, heat, mass transfer rate are given by

$$
\overline{\tau_{L}}=\frac{2 \mu}{L^{2}} \int_{0}^{L} x\left(\frac{\partial u}{\partial y}\right)_{y=0} d x
$$

Heat transfer coefficient is given by

$$
\begin{aligned}
& \bar{h}=\frac{-2 k}{L^{2}} \int_{0}^{L} \frac{x\left(\frac{\partial T^{\prime}}{\partial y}\right)_{y=0}}{T_{w}^{\prime}-T_{\infty}^{\prime}} d x, \\
& \overline{N_{u_{L}}}=\frac{L \bar{h}}{k}=-\frac{2}{L} \int_{0}^{L} \frac{x\left(\frac{\partial T^{\prime}}{\partial y}\right)_{y=0}}{T_{w}^{\prime}-T_{\infty}^{\prime}} d x, \\
& \overline{S_{h_{L}}}=\frac{L k_{1}}{D}=-\frac{2}{L} \int_{0}^{L} \frac{x\left(\frac{\partial C^{\prime}}{\partial y}\right)_{y=0}}{C_{w}^{\prime}-C_{\infty}^{\prime}} d x .
\end{aligned}
$$

Establishing the following non dimensional variables for the parametric study 


$$
\begin{aligned}
& X=\frac{x}{L}, \quad Y=\frac{y}{L}\left(\mathrm{Gr}_{L}\right)^{\frac{1}{4}}, \quad R=\frac{r}{L}, \quad \text { where } \quad r=x \sin \phi, \\
& V=\frac{v L}{v}\left(\mathrm{Gr}_{L}\right)^{\frac{-1}{5}}, \quad U=\frac{u L}{v}\left(\mathrm{Gr}_{L}\right)^{\frac{-2}{5}}, \quad t=\frac{v t^{\prime}}{L^{2}}\left(\mathrm{Gr}_{L}\right)^{\frac{2}{5}}, \\
& T=\frac{T^{\prime}-T_{\infty}^{\prime}}{\left(q_{w}(L) L / k\right)}, \quad \mathrm{Gr}_{L}=\frac{g \beta\left(T_{w}^{\prime}-T_{\infty}^{\prime}\right) L^{3} \cos \phi}{v^{2}}, \quad \operatorname{Pr}=\frac{v}{\alpha}, \\
& C=\frac{\left(C^{\prime}-C_{\infty}^{\prime}\right)}{\left(q_{w}(L) L / D\right)}, \quad \mathrm{Gr}^{*}=\frac{g \beta_{c}\left(C_{w}^{\prime}-C_{\infty}^{\prime}\right) L^{3} \cos \phi}{v^{2}}, \quad \mathrm{Sc}=\frac{v}{D}, \\
& N=\frac{\mathrm{Gr}^{*}}{\mathrm{Gr}_{L}}, \quad \Delta=\frac{Q_{o} L^{2}}{C_{p} \mu}\left(\mathrm{Gr}_{L}\right)^{\frac{-1}{5}}, \quad \lambda=\frac{k_{1} L^{2}}{v}\left(\mathrm{Gr}_{L}\right)^{\frac{-1}{5}}, \quad M=\frac{\sigma B_{0}^{2} L^{2}}{\mu} \mathrm{Gr}_{L}^{-1 / 5}, \\
& R_{d}=\frac{K_{1}^{*} K}{4 \sigma^{*} T_{\infty}^{\prime 3}}, \quad \varepsilon=\frac{g \beta L}{C_{P}},
\end{aligned}
$$

where $\varepsilon$ is the Eckert number as explained by Gebhert [34] and Jordan [35].

The above Eqs (2.1)-(2.5) be able to be written in dimensionless form

$$
\begin{aligned}
& \frac{\partial}{\partial X}(U R)+\frac{\partial}{\partial Y}(V R)=0, \\
& \frac{\partial U}{\partial t}+U \frac{\partial U}{\partial X}+V \frac{\partial U}{\partial Y}-\frac{\partial^{2} U}{\partial Y^{2}}-(T+N C)+M U=0, \\
& \frac{\partial T}{\partial t}+U \frac{\partial T}{\partial X}+V \frac{\partial T}{\partial Y}-\frac{1}{\operatorname{Pr}}\left(1+\frac{4}{3 R_{d}}\right) \frac{\partial^{2} T}{\partial Y^{2}}-\Delta T-\varepsilon\left(\frac{\partial U}{\partial Y}\right)^{2}=0, \\
& \frac{\partial C}{\partial t}+U \frac{\partial C}{\partial X}+V \frac{\partial C}{\partial Y}-\frac{1}{\operatorname{Sc}} \frac{\partial^{2} C}{\partial Y^{2}}+\lambda C=0 .
\end{aligned}
$$

The dimensionless appropriate initial, boundary conditions are

$$
\begin{aligned}
& t \leq 0: U=0, \quad V=0, \quad T=0, C=0 \text { for all } X \text { and } Y, \\
& t>0: \quad U=0, \quad V=0, \quad \frac{\partial T}{\partial Y}=-X^{n}, \frac{\partial C}{\partial Y}=-X^{m} \quad \text { at } Y=0, \\
& U=0, \quad T=0, C=0 \quad \text { at } X=0, \\
& U \rightarrow 0, \quad T \rightarrow 0, \quad C \rightarrow 0 \quad \text { as } \quad Y \rightarrow \infty .
\end{aligned}
$$

Local shear stress, heat and mass transfer rate in dimensionless form are

$$
\tau_{X}=\operatorname{Gr}_{L}^{\frac{3}{5}}\left(\frac{\partial U}{\partial Y}\right)_{Y=0},
$$




$$
\begin{gathered}
\mathrm{Nu}_{X}=\frac{X}{T_{Y=0}}\left(\frac{-\partial T}{\partial Y}\right)_{Y=0} \mathrm{Gr}_{L}^{\frac{1}{5}}, \\
\mathrm{Sh}_{X}=\frac{X}{C_{Y=0}}\left(\frac{-\partial C}{\partial Y}\right)_{Y=0} \mathrm{Gr}_{L}^{\frac{3}{5}} .
\end{gathered}
$$

Dimensionless average skin friction heat and mass transfer rate are

$$
\begin{aligned}
& \bar{\tau}=2 \mathrm{Gr}_{L} \int_{0}^{\frac{3}{4}} \int_{0}^{1} X\left(\frac{\partial U}{\partial Y}\right)_{Y=0} d X, \\
& \overline{\mathrm{Nu}}=2 \mathrm{Gr}_{L}^{\frac{1}{4}} \int_{0}^{1} \frac{X}{T_{Y}=0}\left(\frac{-\partial T}{\partial Y}\right)_{Y=0} d X \\
& \overline{\mathrm{Sh}}=2 \mathrm{Gr}_{L}^{\frac{1}{4}} \int_{0}^{1} \frac{X}{C_{Y}=0}\left(\frac{-\partial C}{\partial Y}\right)_{Y=0} d X
\end{aligned}
$$

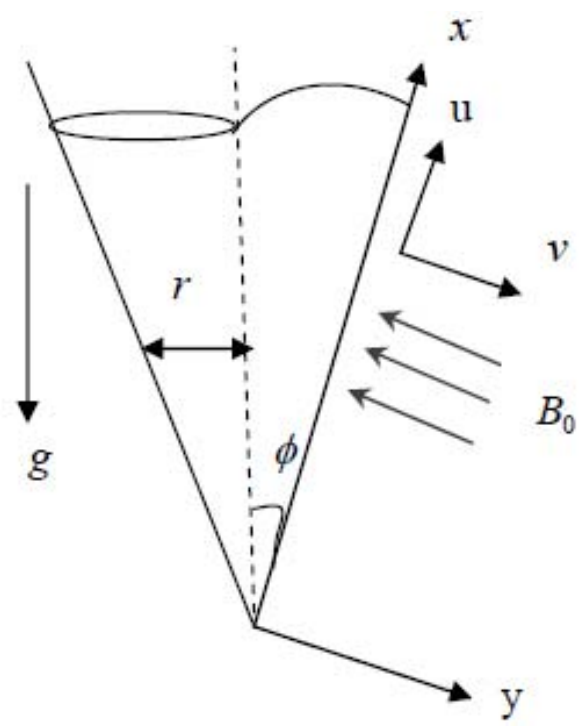

Fig.1. Physical model and coordinate system.

\section{Method of solution}

The finite difference scheme is one of the dependable procedure for solving partial differential equations. Crank-Nicolson method has been adopted to solve Eqs (2.17)-(2.20) with the help of Eq.(2.15). The integral region has been assumed to be a rectangular mesh with, $X \max =1$ and $Y \max =18$, where $Y_{\max }$ $=\infty$ which is farthest from all periphery layers. The assessment of $Y$ max was selected after a few initial examinations, so that the ultimate and penultimate periphery conditions of Eq.(2.15) are contented. The mesh sizes have been predetermined as $\Delta X=0.05, \Delta Y=0.05$ and $\Delta t=0.01$. In this assumption, size of the spatial mesh is decreased by $50 \%$ in $x$ - direction, followed by in $x$ and $y$ directions and the outcomes are analyzed. It is noticed that it is contented with correctness up to the lenience limit of $10^{-5}$. Hence, the mesh sizes adopted here are believed to be suitable for computations. Computations are conceded out until stability is attained. Central difference procedure is adopted for the computing spatial coordinates. The terms in the 
PDE's are transformed to difference equations and the ensuing algebraic problem is deciphered with the help of a well known Thomas algorithm. The differential coefficients associated in Eqs (2.22)-(2.24) are achieved by five point approximation formula then the integrals are assessed using Newton-Cotes formula.

The Crank Nicholson Finite Difference equation equivalent to the equations are specified by

Equation of Momentum

$$
\begin{aligned}
& U_{i, j-1}^{k+1}\left[-V_{i, j}^{k} \frac{\Delta t}{4 \Delta Y}-\frac{\Delta t}{2(\Delta Y)^{2}}\right]+U_{i, j}^{k+1}\left[1+U_{i, j}^{k} \frac{\Delta t}{2 \Delta X}+\frac{\Delta t}{(\Delta Y)^{2}}+M \frac{\Delta t}{2}\right]+U_{i, j+1}^{k+1}\left[V_{i, j}^{k} \frac{\Delta t}{4 \Delta Y}-\frac{\Delta t}{2(\Delta Y)^{2}}\right]+ \\
& -U_{i, j}^{k}+U_{i, j}^{k}\left[\frac{\left(U_{i-1, j}^{k+1}-U_{i, j}^{k}+U_{i-1, j}^{k}\right) \Delta t}{2(\Delta X)}\right]-V_{i, j}^{k}\left[\frac{\left(U_{i, j-1}^{k}-U_{i, j+1}^{k}\right) \Delta t}{4 \Delta Y}\right]+ \\
& -\left[\left\{\left(\frac{T_{i, j}^{k+1}+T_{i, j}^{k}}{2}\right)+N\left(\frac{C_{i, j}^{k+1}+C_{i, j}^{k}}{2}\right)\right\} \Delta t\right] \cos \phi+\left[\left(\frac{U_{i, j-1}^{k}-2 U_{i, j}^{k}+U_{i, j+1}^{k}}{2(\Delta Y)^{2}}\right) \Delta t\right]+M U_{i, j}^{k} \frac{\Delta t}{2} .
\end{aligned}
$$

Equation of energy

$$
\begin{aligned}
& T_{i, j-1}^{k+1}\left[-V_{i, j}^{k} \frac{\Delta t}{4 \Delta Y}-\frac{1}{\operatorname{Pr}}\left(\frac{3 N+4}{3 N}\right) \frac{\Delta t}{2(\Delta Y)^{2}}\right]+T_{i, j}^{k+1}\left[1+U_{i, j}^{k} \frac{\Delta t}{2 \Delta X}+\frac{1}{\operatorname{Pr}}\left(\frac{3 N+4}{3 N}\right) \frac{\Delta t}{(\Delta Y)^{2}}-\Delta\left(\frac{\Delta t}{2}\right)\right]+ \\
& +T_{i, j+1}^{k+1}\left[V_{i, j}^{k} \frac{\Delta t}{4 \Delta Y}-\frac{1}{\operatorname{Pr}}\left(\frac{3 N+4}{3 N}\right) \frac{\Delta t}{2(\Delta Y)^{2}}\right]-T_{i, j}^{k}+U_{i, j}^{k}\left[\frac{\left(T_{i-1, j}^{k+1}+T_{i-l, j}^{k}-T_{i, j}^{k}\right) \Delta t}{2(\Delta X)}\right]+ \\
& -V_{i, j}^{k}\left[\frac{\left(T_{i, j-1}^{k}-T_{i, j+1}^{k}\right) \Delta t}{4 \Delta Y}\right]-\frac{1}{\operatorname{Pr}}\left(\frac{3 N+4}{3 N}\right)\left[\left(T_{i, j-1}^{k}-2 T_{i, j}^{k}+T_{i, j+1}^{k}\right) \frac{\Delta t}{2(\Delta Y)^{2}}\right]+ \\
& -\Delta T_{i, j}^{k}\left(\frac{\Delta t}{2}\right)-\varepsilon\left[\frac{U_{i, j+1}^{k}-U_{i, j}^{k}}{\Delta Y}\right]^{2} .
\end{aligned}
$$

Concentration equation

$$
\begin{aligned}
& C_{i, j-1}^{k+1}\left[V_{i, j}^{k} \frac{\Delta t}{4 \Delta Y}-\frac{1}{\mathrm{Sc}} \frac{\Delta t}{2(\Delta Y)^{2}}\right]+C_{i, j}^{k+1}\left[1+U_{i, j}^{k} \frac{\Delta t}{2 \Delta X}+\frac{1}{\mathrm{Sc}} \frac{\Delta t}{(\Delta Y)^{2}}+\lambda\left(\frac{\Delta t}{2}\right)\right]+ \\
& +C_{i, j+1}^{k+1}\left[V_{i, j}^{k} \frac{\Delta t}{4 \Delta Y}-\frac{1}{\mathrm{Sc}} \frac{\Delta t}{2(\Delta Y)^{2}}\right]-\left(C_{i, j}^{k}-U_{i, j}^{k}\right)\left[\frac{\left(C_{i-l, j}^{k+1}-C_{i-1, j}^{k}+C_{i, j}^{k}\right) \Delta t}{2(\Delta X)}\right]+ \\
& -V_{i, j}^{k}\left[\frac{\left(C_{i, j+1}^{k}-C_{i, j-1}^{k}\right) \Delta t}{4 \Delta Y}\right]-\frac{1}{\mathrm{Sc}}\left[\left(C_{i, j-1}^{k}-2 C_{i, j}^{k}+C_{i, j+1}^{k}\right) \frac{\Delta t}{2(\Delta Y)^{2}}\right]+\lambda C_{i, j}^{k}\left(\frac{\Delta t}{2}\right) .
\end{aligned}
$$

\section{Exchange of views on results}

Representative numerical upshots for the transient heat and mass diffusion in the occurrence of MHD, radiation, heat generation, viscous dissipation and chemical reaction will be discussed and which is the prime idea in this section. 


\section{A. Momentum profiles}

Figures $2 \mathrm{a}, 2 \mathrm{~b}$ and $2 \mathrm{cdepicts}$ the transient velocity profiles for different values of $\mathbf{S c}$, radiation parameter $\boldsymbol{R} \boldsymbol{d}$ viscous dissipation parameter $\varepsilon$ heat generation parameter $\Delta$, chemical reaction parameter $\lambda$ magnetic field parameter $\boldsymbol{M}$. The tendency confirms the role of mass dispersion is highly dominant in momentum field. The effect of distinct values of $\mathrm{Sc}, R d$, and $\varepsilon$ are exhibited in Fig.2a. It is noticed that the momentum rises with reducing $R d$. This demonstrates that, due to the elevated thermal radiation the momentum reduces enormously. The viscous dissipative heat shoots up the momentum near the exterior of the cone. It is also inferred that the momentum diminishes with rising Sc.

The effect of $\Delta$ over momentum is viewed from the Fig.2b, it reveals that the velocity rises for the generative response and reduces for a destructive response. The effect of velocity for distinct chemical reaction parameter and MHD values are exposed in Fig.2c. It is studied that the momentum boosts with diminishing values of $\lambda$ and $\boldsymbol{M}$. This illustrates that the rise in $\boldsymbol{M}$ directs to descend in the momentum. This is because the magnetic field put forth a retarding force on natural convection flow.

\section{B. Temperature profiles}

The temperature summary for dissimilar quantities of $R d, \varepsilon$ and Schmidt number are portrayed in Fig.3a. It is viewed that the temperature amplifies with declining $R d$ and dissipation parameter $\varepsilon$. This demonstrates that the buoyancy upshot on the temperature distribution is most important in air.

It is acknowledged that $R d$ and Pr plays an imperative role in flow phenomenon as, it is a quantity of relative scale of thickness of the boundary layers of viscous to thermal. Lesser temperatures are detected for inferior quantities of Sc. The temperature augments with Schmidt number and MHD is monitored from Fig.3c. Furthermore, we can wind up from Fig.3b that the temperature rises with $\lambda$.

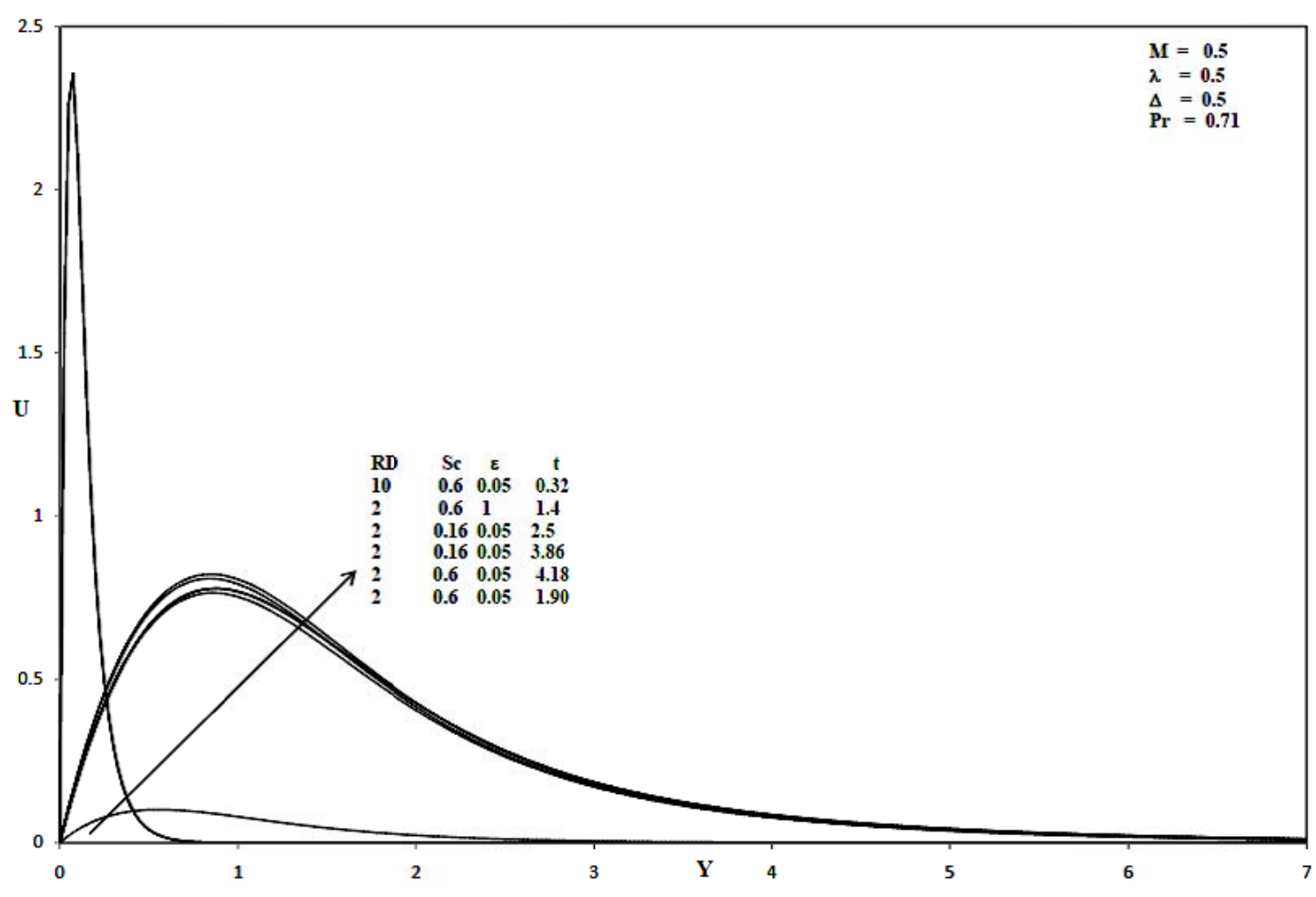

Fig.2a. Transient velocity profiles for various values of $R d, \mathrm{Sc}, \varepsilon$. 


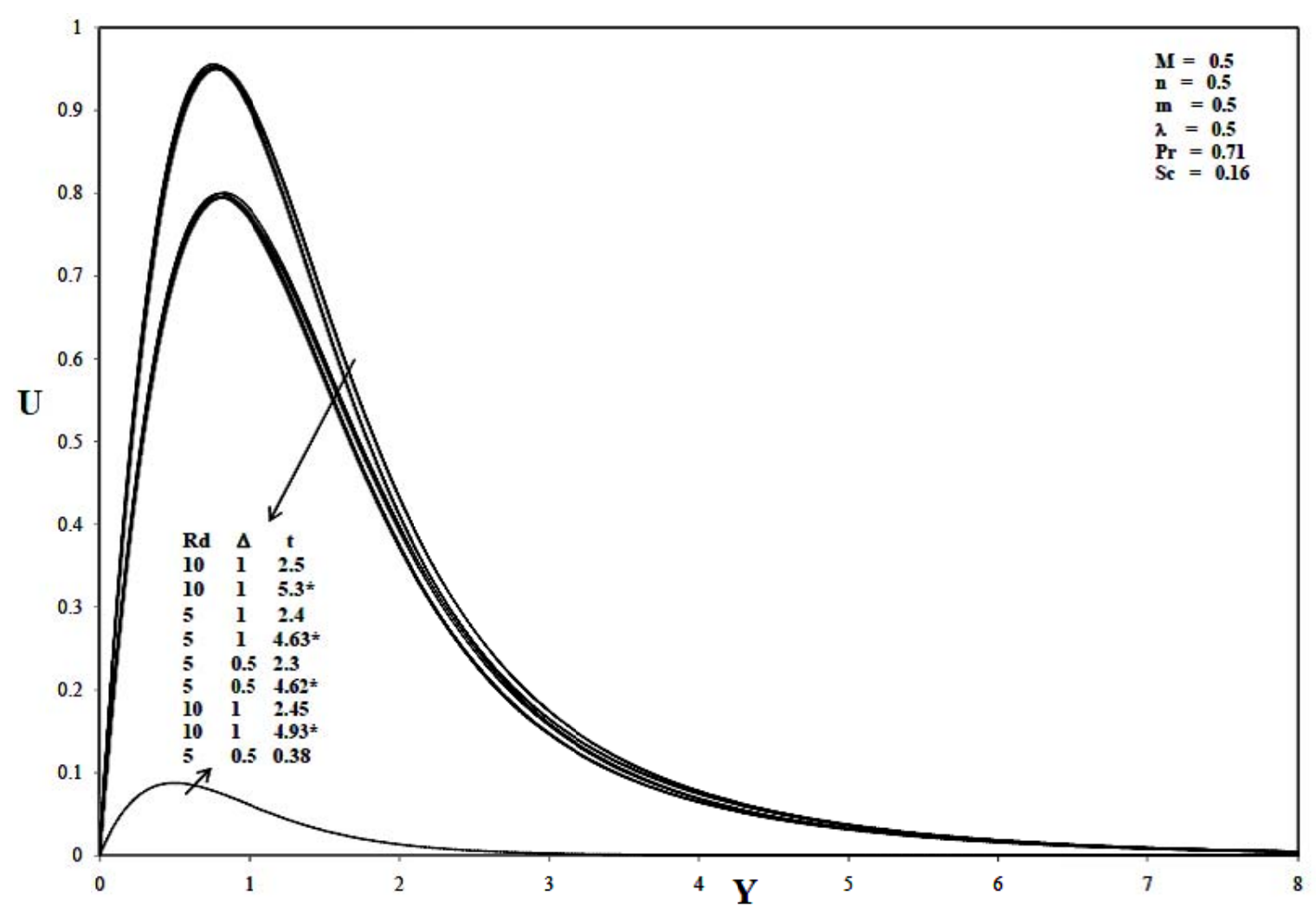

Fig.2b. Unsteady velocity profiles for different values of $R d, \Delta$.

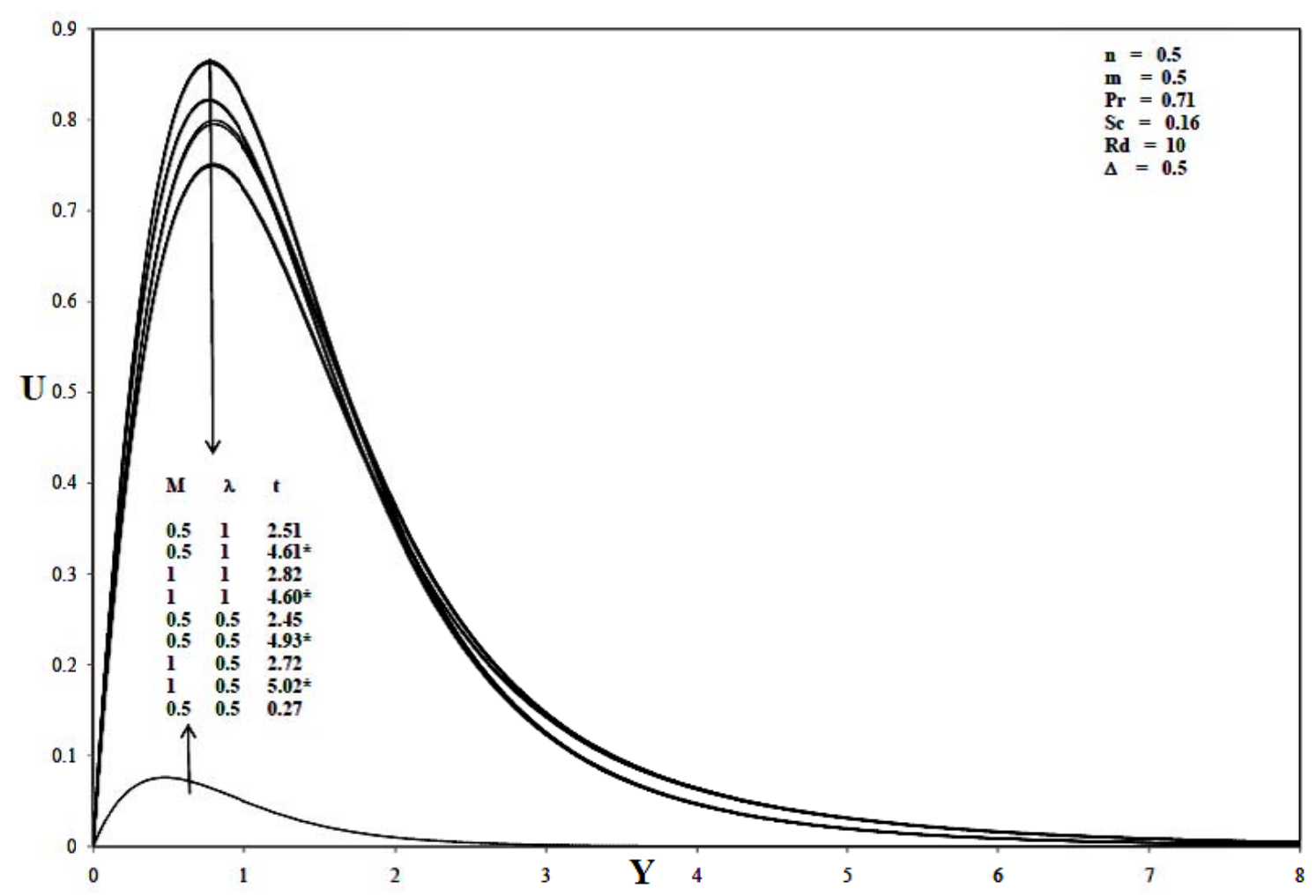

Fig.2c. Unsteady velocity profiles for different values of $\lambda, M$. 


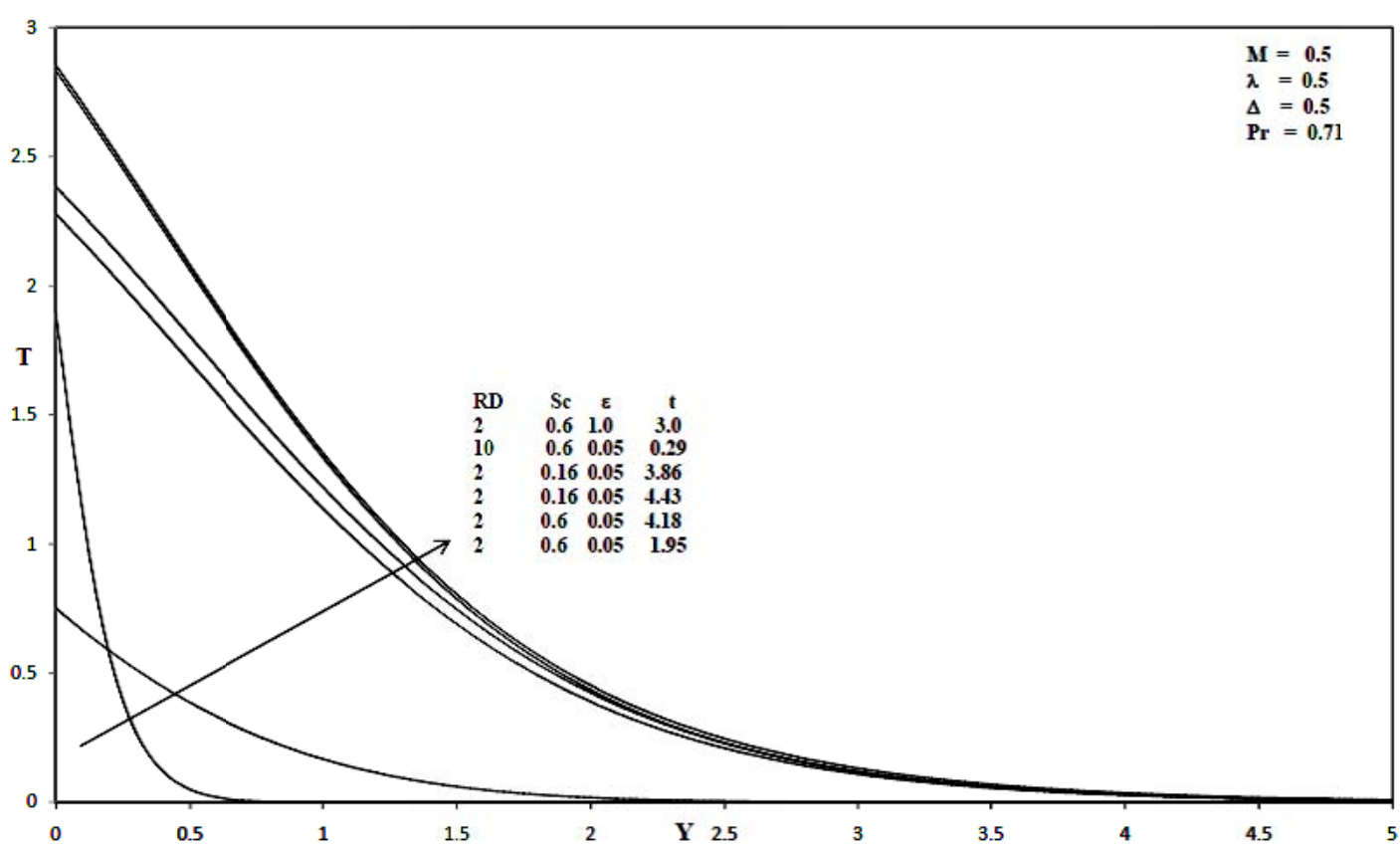

Fig.3a. Unsteady temperature profiles for different values of $R d, \mathrm{Sc}, \varepsilon$.

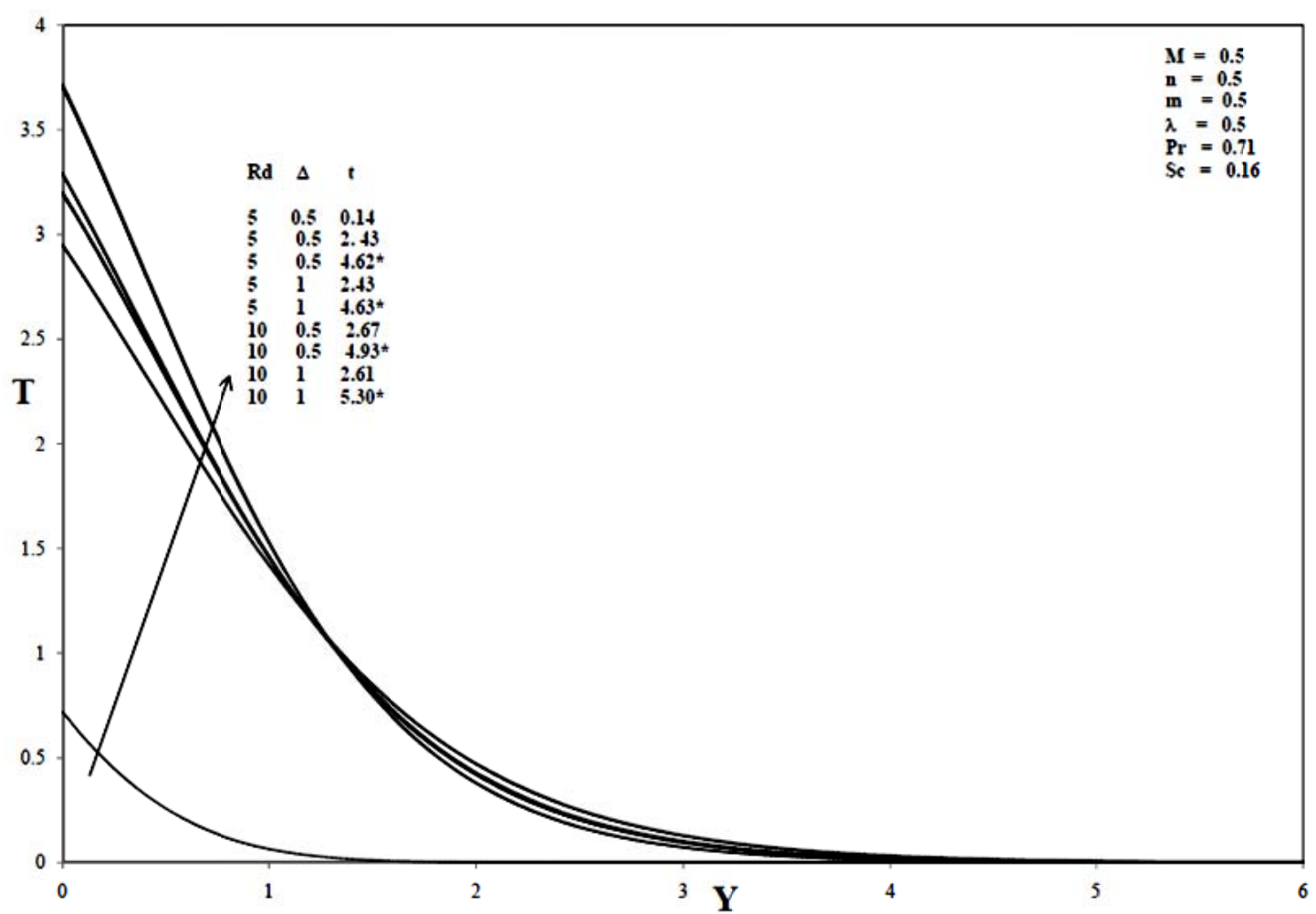

Fig.3b. Unsteady temperature profiles for different values of $R d, \Delta$. 


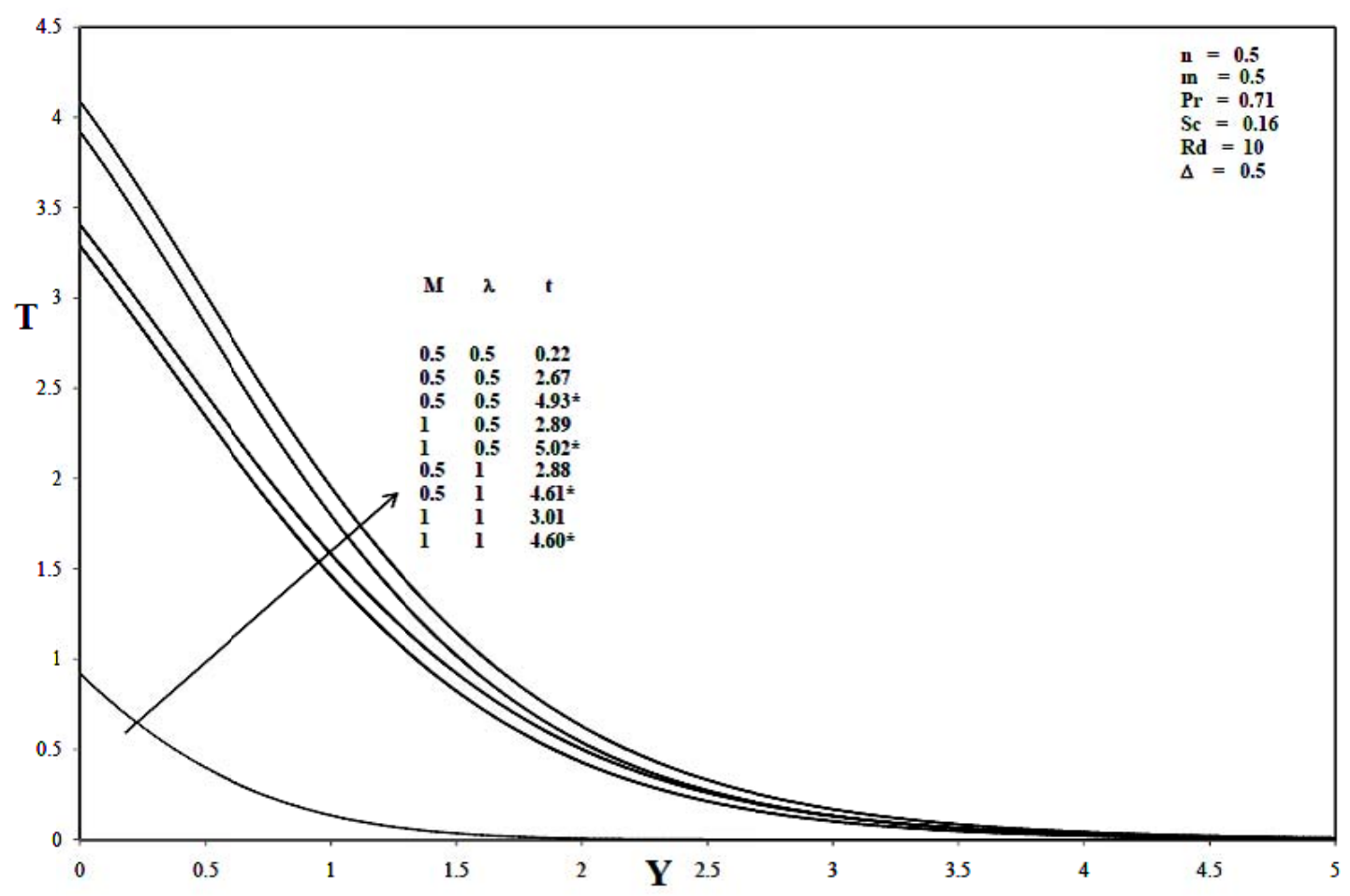

Fig.3c. Unsteady velocity profiles for different values of $\lambda, M$.

\section{Concentration profiles}

Unsteady concentration impacts for distinct quantities of the physical parameters are expressed graphically through Figs $4 \mathrm{a}, 4 \mathrm{~b}$ and $4 \mathrm{c}$. It is noticed that the surface concentration augments with diminishing $R d, \mathrm{Sc}$ and $\varepsilon$. It is perceived that concentration rises with MHD. As anticipated, the species concentration is lesser at elevated quantities of the Sc.

There is a reduction in concentration is viewed by the rising quantities of $R d$ and $\Delta$ is inferred in Fig.4b. Figure 4c illustrates the effects of $\lambda$ and $M$ over the concentration profiles, it reveals that the concentration rises for higher numerical values of $\lambda$ and a smaller amount values of $M$.

\section{Profiles of Shear stress, Nusselt number and Sherwood number}

The local skin friction, Nusselt number and mass transfer rate consequences are plotted in Figs 5, 6 and 7 respectively. The shear stress decelerates with diminishing radiation parameter. The local heat transfer rate increases with rising radiation parameter. The local Sherwood number increases with $\lambda$. For a constructive response, the skin friction increases with escalating numerical values of $\lambda$. The heat transfer rate for distinct numerical values of $R d$ is portrayed in Fig. 6 as a function of the axial co-ordinate. It is observed, heat transfer rate rises with escalating quantities of $R d$. The mass transfer rate for distinct quantities of Sc is illustrated in Fig.7. As anticipated, the Sherwood number rises with mounting quantities of Sc. This tendency is just turn round as contrast to the concentration. 


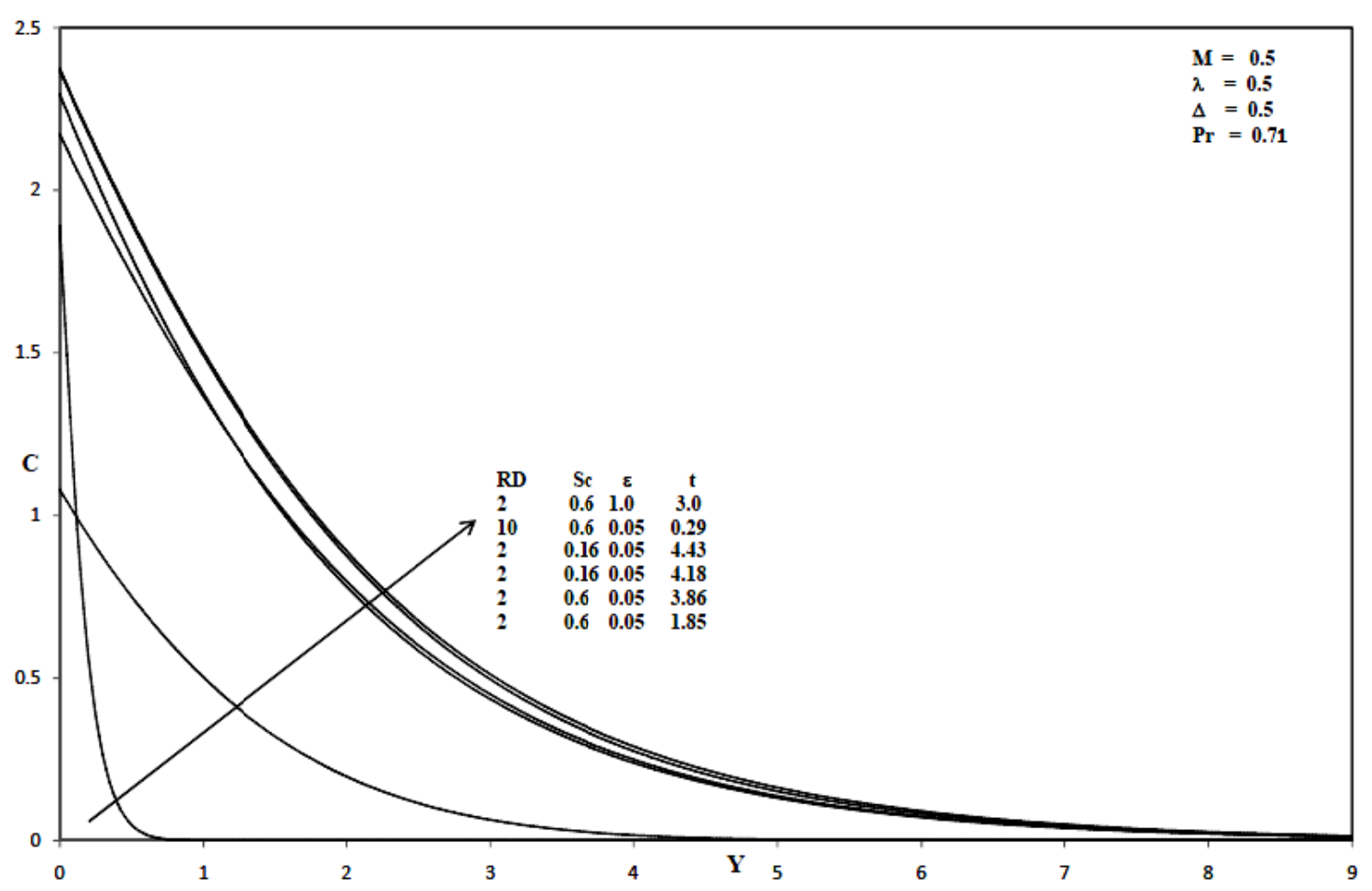

Fig.4a. Unsteady concentration profiles for different values of $R d, \mathrm{Sc}, \varepsilon$.

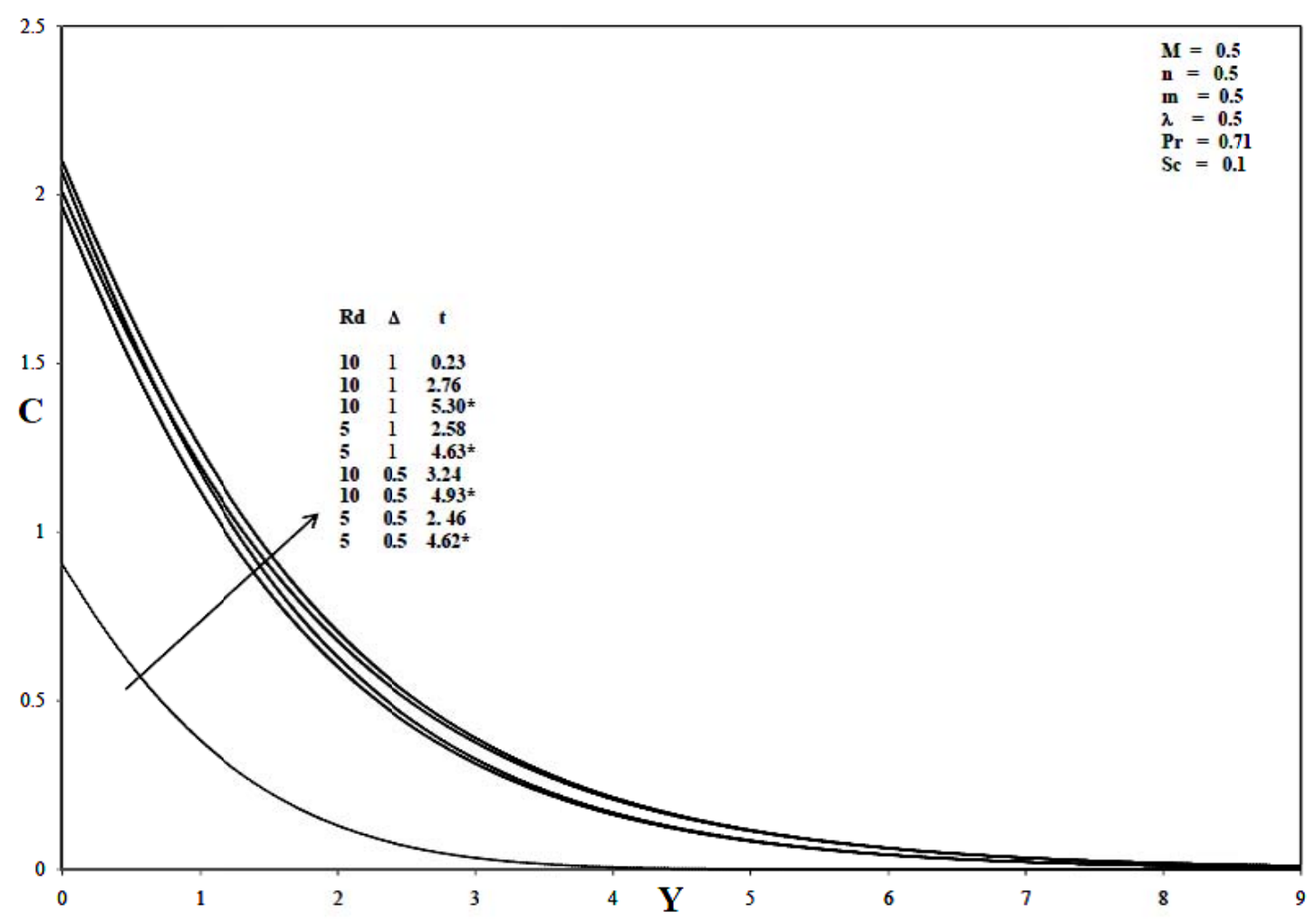

Fig.4b. Unsteady concentration profiles for different values of $R d, \Delta$. 


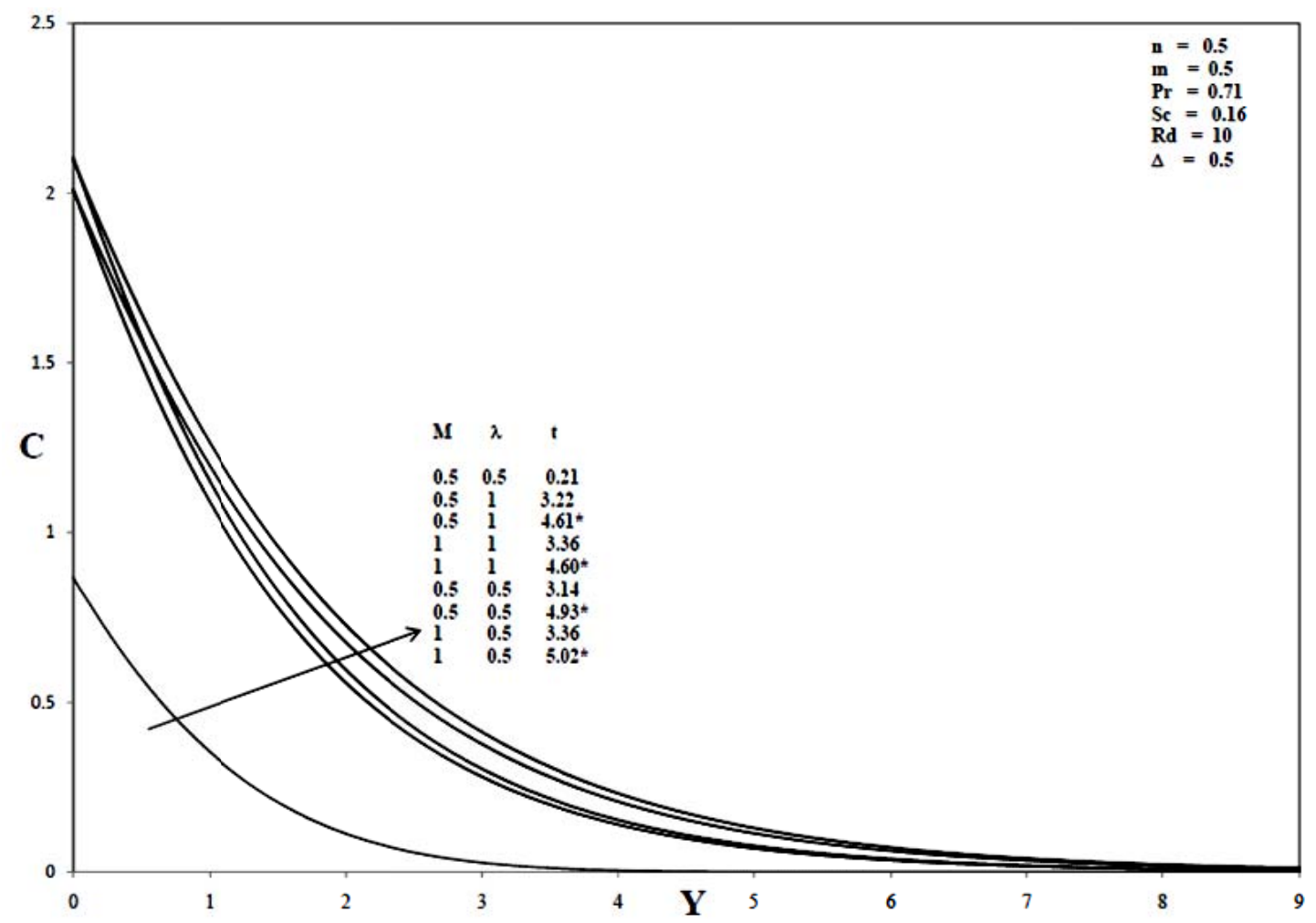

Fig.4c.Unsteady concentration profiles for different values of $\lambda, M$.

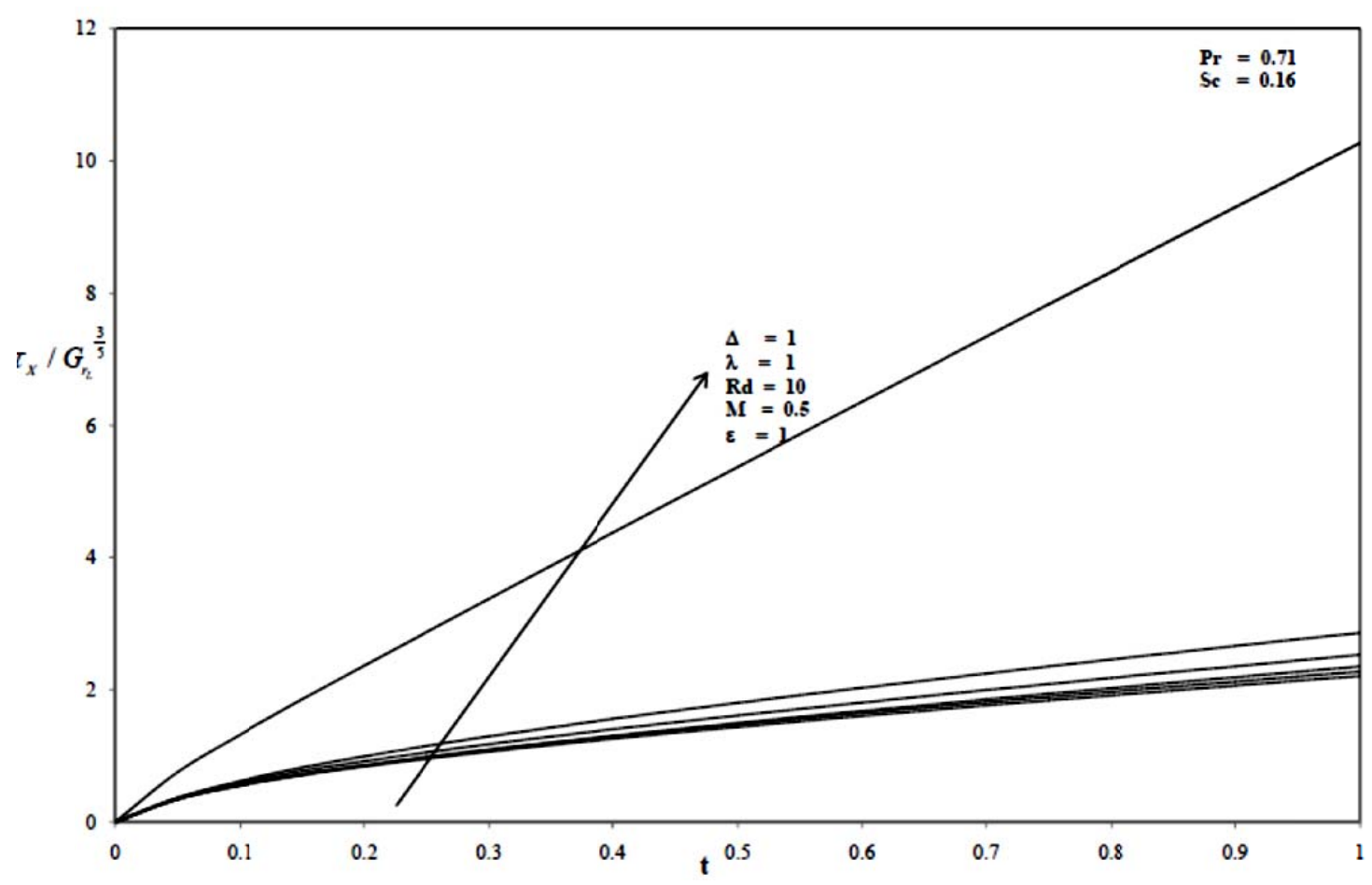

Fig.5. Local skin friction. 


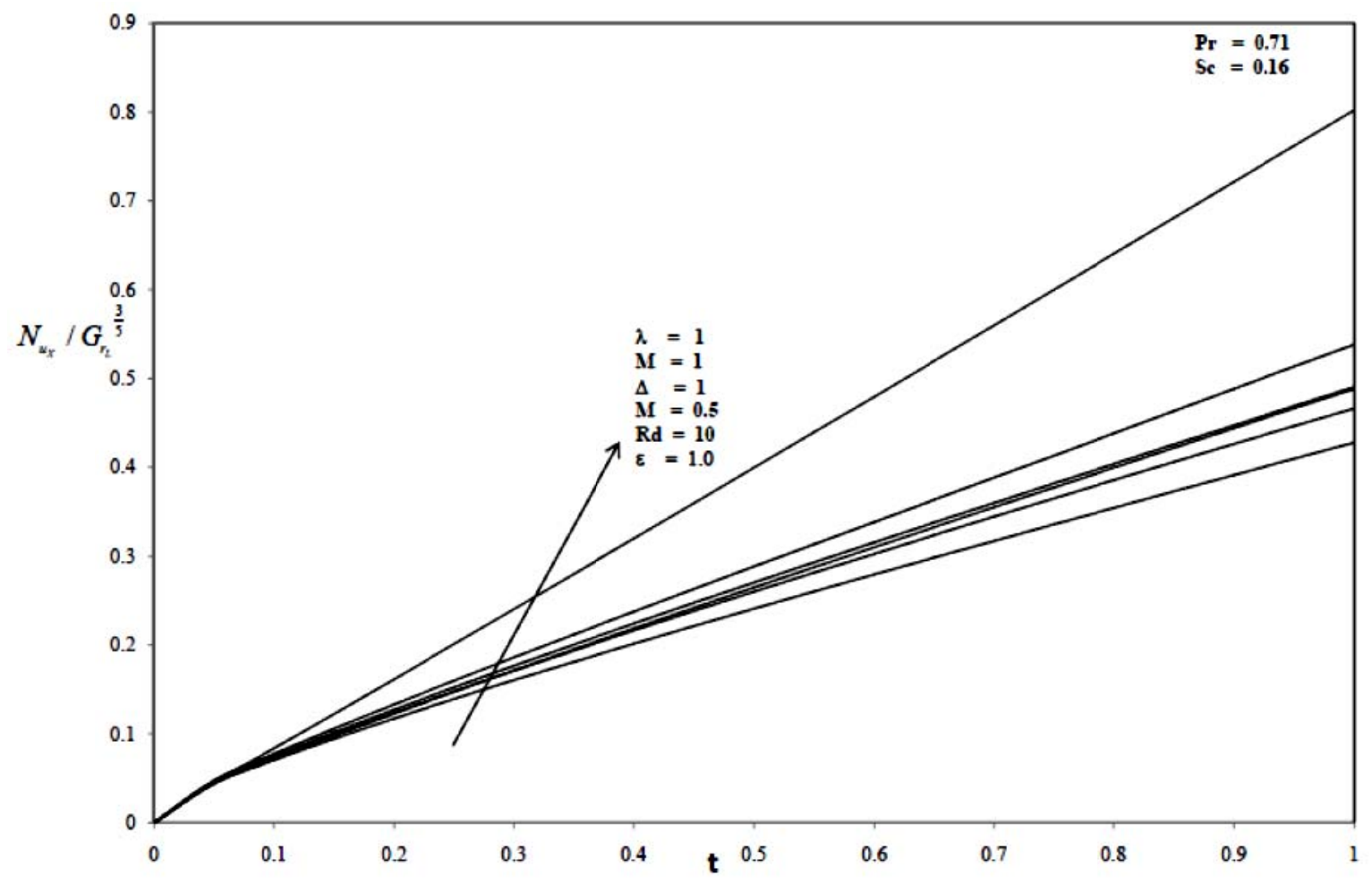

Fig.6. Local Nusselt number.

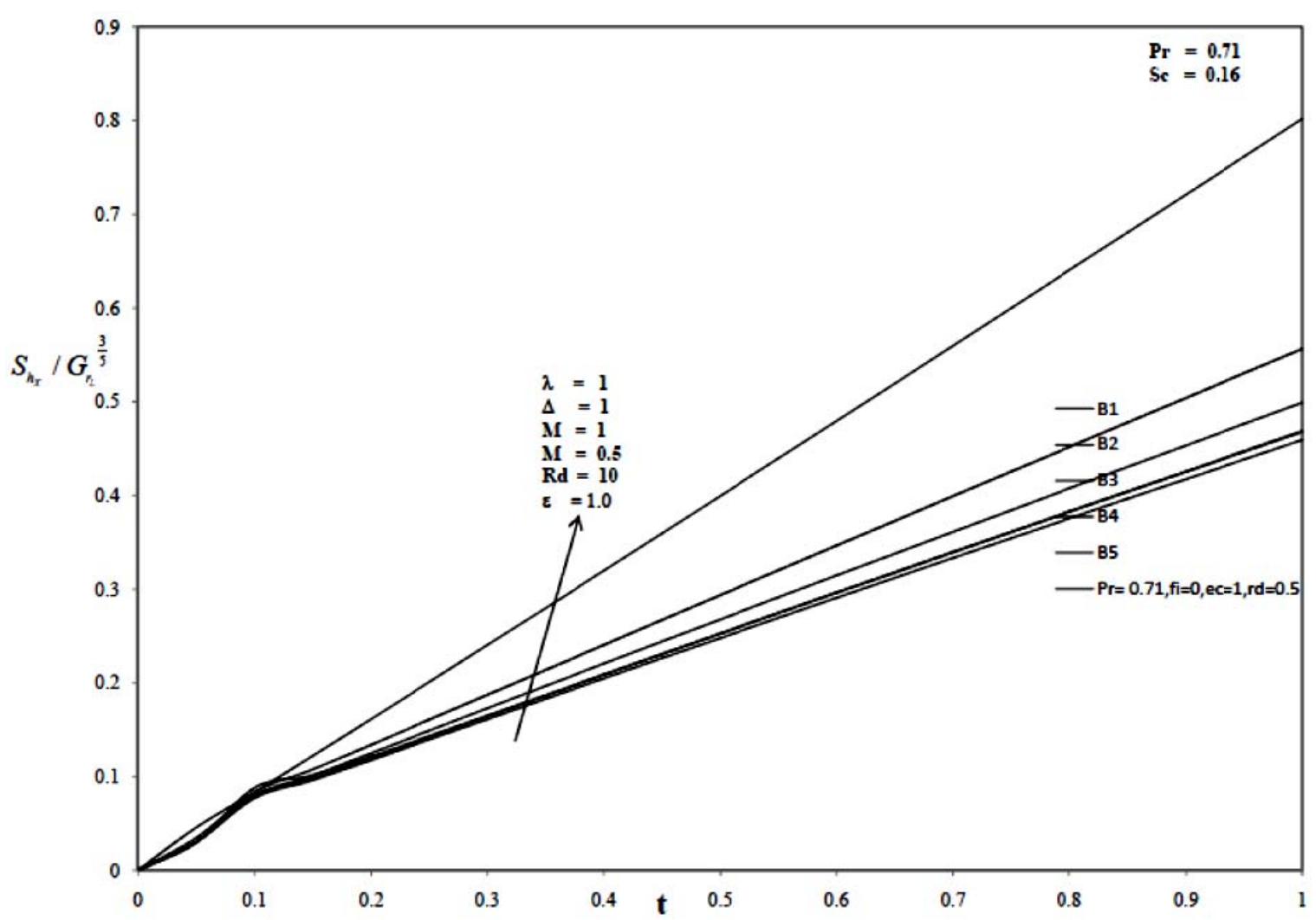

Fig.7. Local Sherwood number. 


\section{Conclusion}

In this work, we have studied the governing equations for the transient radiative MHD heat and mass transfer flow past an upright cone in the existence of a chemical reaction has been formulated and numerical solutions have been derived using Crank -Nicholson method.

- It is noticed momentum of the fluid flow raises with a rise in $R d, \operatorname{Pr}, \varepsilon$.

- Temperature of the fluid decreases with a rise in $\lambda$ and Schmidt number.

- Temperature of the fluid raises with a rise in $\varepsilon$.

- Concentration of the fluid rises with a decrease in the Schmidt number and $\lambda$.

\section{Nomenclature}

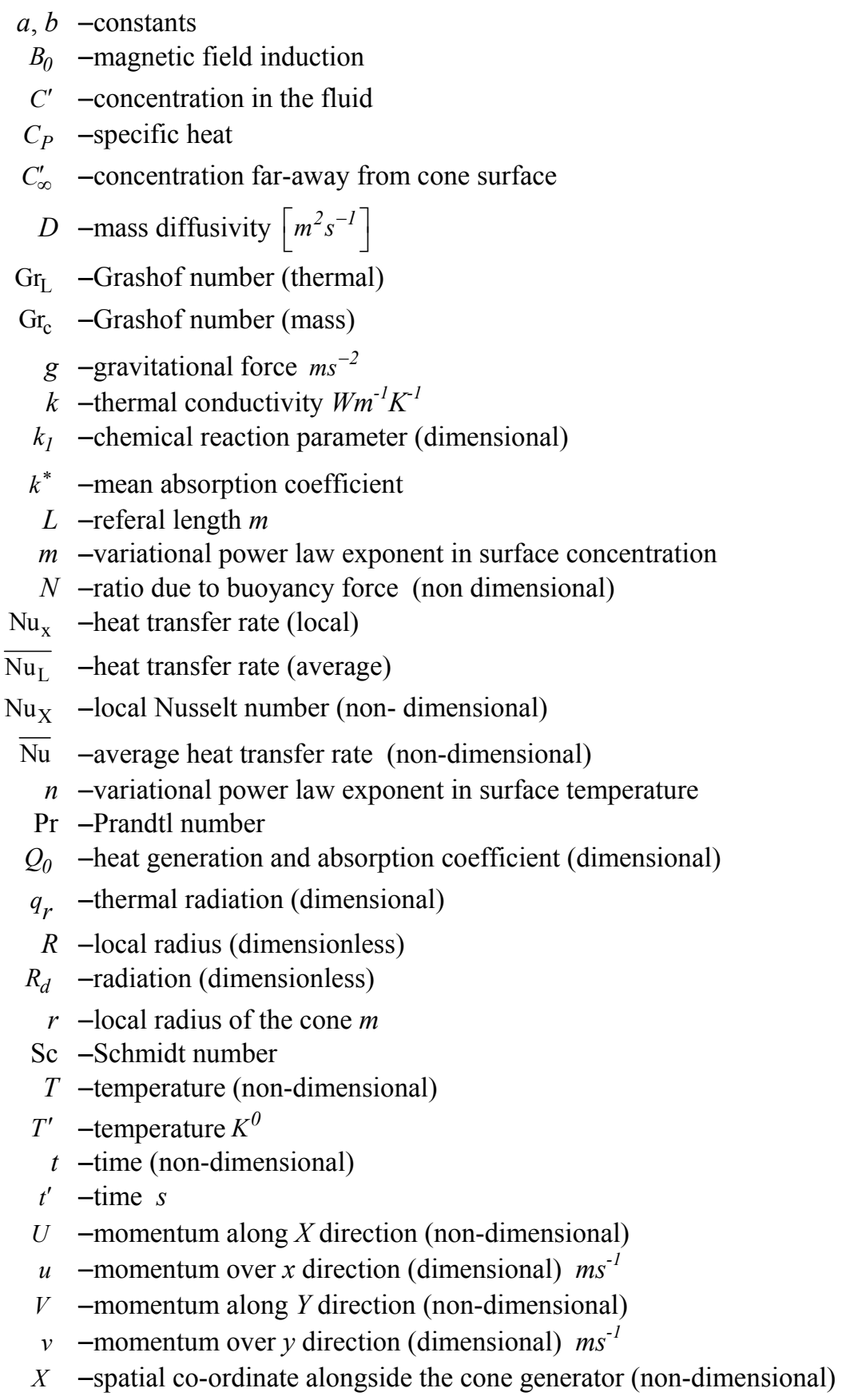




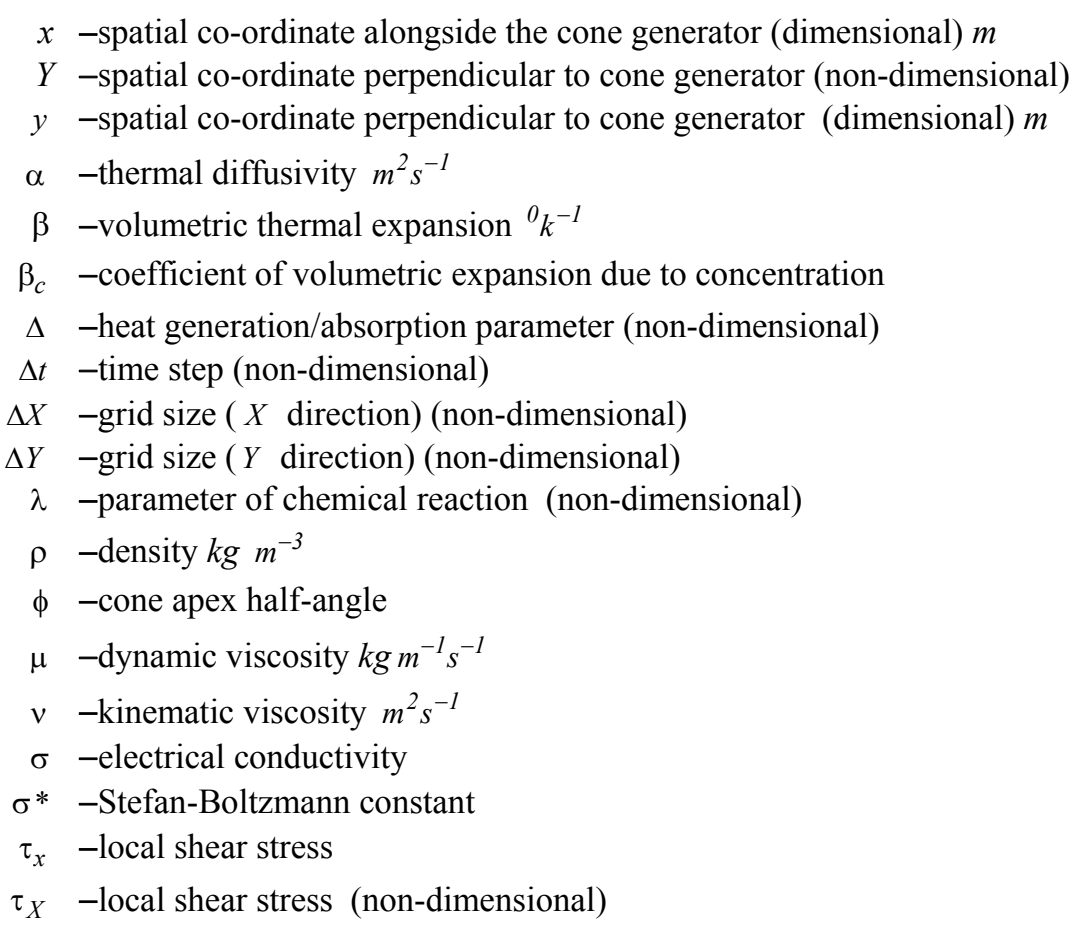

\section{Subscripts}

$w$-wall condition

$\infty \quad$-free-stream condition

\section{References}

[1] Rao J.A. and Shivaiah S. (2011): Chemical reaction effects on unsteady MHD flow past semi-infinite vertical porous plate with viscous dissipation. - Meccanica, vol.32, No.8, pp.1065-1078.

[2] Siddiqa S., Asghar S. and Hossain Md. A. (2010): Radiation effects in mixed convection flow of a viscous fluid having temperature-dependent density along a permeable vertical plate. - Applied Mathematics and Mechanics, vol.31, No.10, pp.1217-1230.

[3] Srinivasa A.H. and Eswara A.T. (2010): Unsteady mixed convection flow from a moving vertical plate in a parallel free stream: Influence of heat generation or absorption. - International Journal of Heat and Mass Transfer, vol.53, No.21-22, pp.4749-4756.

[4] Ramli N., Ahmad S. and Pop I (2018): MHD forced convection flow and heat transfer of ferro fluids over a moving at plate with uniform heat flux and second-order slip effects. - Scientia Iranica,vol.25, No.4, pp.21862197.

[5] Lakshmi Narayana P.A. and Sibanda P. (2010):Soret and Dufour effects on free convection along a vertical wavy surface in a fluid saturated Darcy porous medium. - International Journal of Heat and Mass Transfer, vol.53, No.15-16, pp.3030-3034.

[6] Kumari M. and Nath G. (2012): Natural convection on a horizontal cone in a porous medium with non-uniform wall temperature/concentration or heat/mass flux and suction/injection. - European Journal of Physics, vol.10, No.5, pp.1150-1167.

[7] Dulal Pal and Talukdar B. (2013): Perturbation technique for unsteady MHD mixed convection periodic flow, heat and mass transfer in micropolar fluid with chemical reaction in the presence of thermal radiation. - Heat Mass Transfer,vol.49, No.2, pp.207-217.

[8] Ullaha H., Islam S., Khan I., Sharidan S. and Fiza M. (2017): MHD boundary layer flow of an incompressible upper-convected Maxwell fluid by optimal homotopy asymptotic method. - Scientia Iranica, vol.24, No.1, pp.202210. 
[9] Pullepu B. and Sambath P. (2014): Free convection flow of dissipative fluid past from non-isothermal vertical cone. - Journal of Engineering Physics Thermo Physics, vol.87, No.4, pp.962-972.

[10] Pullepu B., Sambath P. and Viswanathan K.K. (2014): Effects of chemical reactions on unsteady free convective and mass transfer flow from a vertical cone with heat generation/absorption in the presence of VWT/VWC. Mathematical Problems in Engineering (Springer), vol.2014, pp.1-20.

[11] Pullepu B., Sambath P., Selva Rani M., Chamkha A.J. and Viswanathan K.K. (2016): Numerical solutions of free convective flow from a vertical cone with mass transfer under the influence of chemical reaction and heat generation/absorption in the presence of UWT/UWC. - Journal of Applied Fluid Mechanics, vol.9, No.1, pp.343357.

[12] Sambath P., Pullepu B., Hussain T. and Sabir Ali Shehzad (2018): Radiated chemical reaction impacts on natural convective MHD mass transfer flow induced by a vertical cone. - Results in Physics(Elsevier),vol.8, pp.304-315.

[13] Muhaimin I., Kandasamy R. and Khamis Azme B. (2012): Numerical investigation of variable viscosities and thermal stratification effects on MHD mixed convective heat and mass transfer past a porous wedge in the presence of a chemical reaction. - Meccanica, vol.47, No.4, pp.863-876.

[14] Zueco J. and Ahmed S. (2013): Combined heat and mass transfer by mixed convection MHD flow along a porous plate with chemical reaction in presence of heat source. - Meccanica,vol.48, No.4, pp.931-942.

[15] Anjali Devi S.P. and Vasantha Kumari D. (2014): Numerical investigation of slip flow effects on unsteady hydromagnetic flow over a stretching surface with thermal radiation. - International Journal of Advanced and Applied Mathematics and Mechanics, vol.1, No.4, pp.20-32.

[16] Alam M.S., Haque M.M. and Uddin M.J. (2014): Unsteady MHD free convective heat transfer flow along a vertical porous flat plate with internal heat generation. - International Journal of Advanced and Applied Mathematics and Mechanics, vol.2, No.2, pp.52-61.

[17] Awad F.G., Sibanda P., Motsa S.S. and Makinde O.D. (2010): Double diffusion from a vertical truncated cone in a non-Newtonian fluid saturated porous medium with variable heat and mass fluxes. - International Communications of Heat Mass Transfer, vol.37, No.3, pp.261-265.

[18] Ching-Yang Cheng (2012): Radiation effects on transient magneto hydrodynamic natural convection flow with heat generation. - International Journal of Thermal Sciences, vol.58, pp.79-91.

[19] Khan W.A., Khan Z.H. and Haq R.U. (2015): Flow and heat transfer of ferrofluids over a at plate with uniform heat flux. - European Physics Journal Plus, vol.130, No.86, 10 pages.

[20] Das S., Jana R.N. and Makinde O.D. (2016): Magneto hydrodynamic free convective flow of nanofluids past an oscillating porous at plate in a rotating system with thermal radiation and hall effects. - Journal of Mechanics, vol.32, No.02, pp.197-210.

[21] Kasmani R.M., Sivasankaran S., Bhuvaneswari M. and Siri Z. (2016): Effect of chemical reaction on convective heat transfer of boundary layer flow in nanofluid over a wedge with heat generation/absorption and suction. Journal of Applied Fluid Mechanics, vol.9, No.1, pp.379-388.

[22] Niranjan H., Sivasankaran S. and Bhuvaneswari M.(2017): Chemical reaction Soret and Dufour effects on MHD mixed convection stagnation- point flow in a porous medium with convective boundary condition. - Int. J. Numerical Methods Heat and Fluid Flow, vol.27, No.2, pp.454-470.

[23] Niranjan H., Sivasankaran S. and Bhuvaneswari M. (2016): Analytical and numerical study on magneto convection stagnation point flow in a porous medium with chemical reaction, radiation and slip effects. Mathematical Problems in Engineering, 2016 Article Id 4017076, pp.1-12.

[24] Chamkha A.J., Abbasbandy S. and Rashad A.M. (2015): Non-Darcy natural convection flow of non-Newtonian nanofluid over a cone saturated in a porous medium with uniform heat and volume fraction fluxes. - International Journal of Numerical Methods and Heat Fluid Flow, vol.25 pp.422-437.

[25] AnuarIshak, Yacob N.A., Nazar R. and Pop I. (2012): Similarity solutions for the mixed convection flow over a vertical plate with thermal radiation. - Journal of Engineering Physics Thermophysics, vol.85, No.2, pp.339-348. 
[26] Crank J. and Nicolson P. (1947): A practical method for numerical evaluation of solutions of partial differential equations of the heat conduction type. - Proceedings of Cambridge. Philosophical Society, vol.43, pp.50-67.

[27] Carnahan B., Luther H.A. and Wilkes J.O. (1969): Applied Numerical Methods. - New York: John Wiley and Sons.

[28] Ruhaila, Kasmani Md., Sivasankaran S., Bhuvaneswari M. and Ahmed Kadhim Hussein (2017): Analytical and numerical study on convection of nanofluid past a moving wedge with Soret and Dufour effects. - Int. J. Numerical Methods Heat and Fluid Flow, vol.27, No.10, pp.2333-2354.

[29] Ramly N.A., Sivasankaran S. and Noor N.F.M. (2017): Zero and non zero normal fluxes of thermal radiative boundary layer flow of nanofluidover a radially stretched surface. - Scientia Iranica, Trans. B. Mech. Engg, vol.24, No.6, pp.2895-2903.

[30] Hsiao K. (2017): Combined electrical MHD heat transfer thermal extrusion system using Maxwell fluid with radiative and viscous dissipation effects. - Applied Thermal Engineering, vol.112, pp.1281-1288.

[31] Pal D. and Mandal G. (2017): Thermal radiation and MHD effects on boundary layer flow of micro polar nanofluid past a stretching sheet with non-uniform heat source/sink. - International Journal of Mechanical Sciences, vol.126, pp.308-318.

[32] Hayat T., Qayyum S., Shehzad S.A. and Alsaedi A. (2017):Simultaneous effects of heat generation/absorption and thermal radiation in magneto hydrodynamics flow of Maxwell nanofluid towards a stretched surface. Results in Physics, vol.7, pp.562-573.

[33] Hayat T., Ijaz Khan M., Farooq M., Alsaedi A. and Imran Khan M. (2017): Thermally stratified stretching flow with Cattaneo-Christov heat flux. - International Journal of Heat and Mass Transfer, vol.106, pp.289-294.

[34] Gebhart B. (1962): Effects of viscous dissipation in natural convection. - Journal of FluidMechanics, vol.14, pp. 225-232.

[35] Zueco Jorda'n J. (2006): Numerical study of an unsteady free convective magneto hydrodynamic flow of a dissipative fluid along a vertical plate subject to a constant heat flux. - International Journal of Engineering Science, vol.44, pp.1380-1393.

[36] Sravanthi C.S. (2018): Slip flow of nanouid over a stretching vertical cylinder in the presence of non-linear thermal radiation and non-uniform heat source/sink. - Scientia Iranica, vol.25, No.4, pp.2098-2110.

[37] Ghaffar Pasand O. (2018): Unsteady double-diffusive natural convection with Soret and Dufour effects inside a two-sided lid-driven skewed enclosure in the presence of applied magnetic field. - Scientia Iranica, vol.25, No.3, pp.1215- 1235 .

[38] Niranjan H., Sivasankaran S. and Bhuvaneswari M. (2017): Chemical reaction radiation and slip effects on MHD mixed convection stagnation- point flow in a porous medium with radiation and slip condition. - Int. J. Numerical Methods Heat and Fluid Flow, vol.24, No.1, pp.698-706.

[39] Loganathan K., Sivasankaran S., Bhuvaneswari M. and Rajan S. (2019): Second-order slip, cross-diffusion and chemical reaction effects on magneto-convection of Oldroyd-B liquid using Cattaneo-Christov heat flux with convective heating. - Journal of Thermal Analysis and Calorimetry, vol.136, No.1, pp.401-409.

[40] Brewster M.Q. (1992): Thermal Radiative Transfer and Properties. - New York: John Wiley and Sons.

Received: May 7, 2019

Revised: August 8, 2019 